\title{
Las vacunas contra la COVID-19, ¿cuál es la mejor?
}

\author{
The vaccines against COVID-19 which is the best? \\ González-Ortiz, José Alfredo; Orihuela-López, Diego Ariel; Xibille- \\ Friedmann, Daniel Xavier
}

\section{José Alfredo González-Ortiz}

Universidad Autónoma del Estado de Morelos, México

Diego Ariel Orihuela-López

Universidad Autónoma del Estado de Morelos, México

\section{Daniel Xavier Xibille-Friedmann}

daniel.xibille@uaem.mx

Universidad Autónoma del Estado de Morelos, México

\author{
Lux Médica \\ Universidad Autónoma de Aguascalientes, México \\ ISSN: 2007-1655 \\ Periodicidad: Cuatrimestral \\ vol. 17, núm. 49,2022 \\ luxmedica.editorial@gmail.com \\ Recepción: 13 Marzo 2021 \\ Aprobación: 29 Septiembre 2021 \\ URL: \\ https://revistas.uaa.mx/index.php/luxmedica/article/view/3129/ \\ DOI: \\ https://doi.org/10.33064/491m20223129
}

\section{c) (i) (2)}

Esta obra está bajo una Licencia Creative Commons AtribuciónNoComercial-CompartirIgual 4.0 Internacional.
Resumen: Introducción: Paralelamente al comienzo de la pandemia por la COVID-19, inició la carrera por desarrollar una vacuna. Actualmente, múltiples vacunas han sido aprobadas por organismos reguladores. Objetivo: Evaluar las diferentes vacunas aprobadas por la COFEPRIS, FDA y EMA, para cumplir con el propósito de responder al cuestionamiento: ¿Cuál es la mejor vacuna contra la COVID-19? Métodos: Se realizó una revisión de la literatura publicada, hasta la primera mitad de marzo del 2021, en la base de datos en línea PubMed y el motor de búsqueda Google Académico. Resultados: Se consultaron los resultados arrojados por cada vacuna en los ensayos clínicos en sus diferentes fases. Se compararon los resultados en términos de eficacia, seguridad, cobertura poblacional, temperatura de conservación, número de dosis requeridas, duración de la respuesta inmune, protección contra variantes, vías de administración y precio. Cada vacuna posee características particulares que las diferencia de las otras y que las acerca a ser la vacuna ideal contra la COVID-19. Conclusión: Determinar que una vacuna es mejor que otra dependerá ampliamente del contexto o término bajo el cual se evalué. De este modo, la mejor vacuna en términos de eficacia es la BNT162b2; en seguridad podría ser Gam-COVID-Vac; en dosis requeridas, la $\mathrm{Ad} 5-\mathrm{nCoV}$, y en precio la $\mathrm{Ad} 5-\mathrm{nCoV}$, o bien la ChAdOx1 nCoV-19. Actualmente, no existe una vacuna ideal contra la COVID-19, no obstante, existen muy buenas opciones en el mercado.

Palabras clave: vacuna, SARS-CoV-2, COVID-19, eficacia, reacciones adversas.

Abstract: Introduction: Parallel to the beginning of the COVID-19 pandemic, the race to develop a vaccine began. Currently multiple vaccines have been approved by regulatory bodies. Objective: Evaluate the different vaccines approved by COFEPRIS, FDA and EMA, to fulfill the purpose of answering the question, which is the best vaccine against COVID-19? Methods: A review of the published literature was carried out, up to the first half of March 2021, in the PubMed online database and the Google Academic search engine. Results: The results obtained by each vaccine in the clinical trials in its different phases were consulted. The results were compared in terms of efficacy, safety, population coverage, storage temperature, number of doses required, duration of the immune response, protection against variants, routes of administration and price. Each vaccine has particular characteristics that differentiate it from the others and that make it the ideal vaccine against COVID-19. Conclusion: 


\begin{abstract}
Determining that one vaccine is better than another will largely depend on the context or term under which it is evaluated. Thus, the best vaccine in terms of efficacy is BNT162b2, in safety it could be Gam-COVID-Vac, in required doses $\mathrm{Ad} 5-\mathrm{nCoV}$ and in price Ad5- $\mathrm{nCoV}$ or ChAdOx1 $\mathrm{nCoV}-19$. Currently there is no ideal vaccine against COVID-19, however there are very good options on the market.
\end{abstract}

Keywords: Vaccine, SARS-CoV-2, COVID-19, efficacy, adverse reactions.

\title{
Introducción
}

La actual pandemia por la COVID-19 comenzó en diciembre de 2019 en Wuhan, China, pero rápidamente trascendió a diversas naciones, entre ellas, México. Para la primera mitad de marzo de 2021, esta enfermedad ha provocado la muerte de más de 2.6 millones de seres humanos en el mundo y se han confirmado aproximadamente 116 millones de casos, y ambas cifras siguen aumentando. ${ }^{1,2}$

Históricamente, después de la potabilización del agua, las vacunas son la herramienta que ha salvado más vidas y el método más eficaz para prevenir enfermedades infecciosas. Debido a esto, paralelamente al comienzo de la pandemia, inició la carrera en búsqueda de una vacuna contra la COVID-19, provocada por el nuevo coronavirus SARS-CoV-2. ${ }^{1,2}$

Para la primera mitad de marzo del 2021, se han desarrollado aproximadamente 200 vacunas contra la COVID-19, de las cuales solo 21 están en fase III de ensayos clínicos. De estas, solamente 11 fueron aprobadas por alguna agencia reguladora en algún país del mundo. En México, la COFEPRIS aprobó cinco vacunas contra la COVID-19: BNT162b2, ChAdOx1 nCoV-19, Ad5-nCoV, Coronavac y Gam-COVID-Vac. Mientras tanto, la FDA en Estados Unidos ha aprobado tres: Ad26.COV2.S, mRNA-1273 y BNT162b2. Igualmente, la EMA en la Unión Europea ha aprobado tres: BNT162b2, ChAdOx1 nCoV-19 y mRNA-1273. A pesar de que todas estas fueron diseñadas para prevenir la infección por SARS-CoV-2, cada una tiene características muy particulares; debido a esto ha surgido un cuestionamiento en la población científica, en el sector salud y en la población en general: ¿Cuál es la mejor vacuna contra la COVID-19? ${ }^{3}$

Para que una vacuna pueda ser considerada ideal para hacer frente a la pandemia de la COVID-19 debe cumplir ciertos criterios: $:^{4,5,6}$

a) Alto grado de eficacia: Igual o mayor al 50\% de eficacia.

b) Alto grado de seguridad: no producir reacciones adversas graves, que comprometan la vida o dejen secuelas irreversibles.

c) Respuesta inmune duradera: de mínimo seis meses.

d) Amplia cobertura poblacional: capaz de administrarse en todo tipo de población desde neonatos, lactantes, niños, adolescentes, adultos, adultos mayores, embarazadas, personas con comorbilidades, inmunocomprometidos, etc. 
e) Facilidad de distribución: debe ser termoestable, capaz de transportarse a temperatura ambiente o en refrigeradores convencionales $\left(2^{\circ} \mathrm{C}\right.$ a $\left.8^{\circ} \mathrm{C}\right)$.

f) Brindar protección contra variantes del antígeno: proteger frente a nuevas variantes del SARS-CoV-2; variante de Reino Unido, sudafricana y brasileña.Brindar protección contra variantes del antígeno: Proteger frente a nuevas variantes del SARS-CoV-2; variante de Reino Unido, sudafricana y brasileña.

g) Monodosis: aplicarse en una sola dosis.

h) Precio accesible: asequible para el grueso de la población.

i) Vía de administración por conjuntivas: administrarse por vía oral, ocular o por mucosas.

El objetivo de este artículo fue evaluar, con base en los criterios antes mencionados, las diferentes vacunas aprobadas por la COFEPRIS, la FDA y la EMA a fin de cumplir con el propósito de responder al cuestionamiento: ¿Cuál es la mejor vacuna contra la COVID-19? La información utilizada para el presente artículo tiene como fecha de corte la primera mitad de marzo de 2021.

\section{Estructura del SARS-CoV-2}

Existen 39 especies de coronavirus, divididas en cinco géneros, 27 subgéneros, y dos subfamilias encasilladas en la familia Coronaviridae. Esta familia se subdividde en cuatro géneros: Alphacoronavirus, Betacoronavirus, Gammacoroavirus y Deltacoronavirus. Los dos primeros se relacionan con infección de mamíferos y los restantes con infección en aves. ${ }^{7,8,9}$

SARS-CoV-2 es un Betacoronavirus del subgénero Sarbecovirus, subfamilia Orthocoronavirinae. Su estructura es esférica, con diámetro de 60 a $140 \mathrm{~nm}$. Su material genético está dispuesto en ácido ribonucleico (ARN) de cadena sencilla en sentido positivo, el cual expresa cuatro de sus principales proteínas estructurales, siendo la nucleocápside la única encontrada en el interior, encargándose de proteger el ARN genómico. En la superficie se encuentran incrustadas proteínas de membrana $(\mathrm{M})$, proteínas de envoltura $(\mathrm{E})$ y proteínas espiga (S). Las primeras ayudan en la formación de viriones intracelulares, así como en el montaje y la generación de envolventes virales maduros. En las segundas tienen lugar los canales iónicos, además de coadyuvar en la secreción de viriones nuevos, teniendo una gran relevancia en la infección. Y las terceras permiten el reconocimiento y la unión a enzimas de la célula huésped, presentando dos subunidades: S1 y S2. La primera contiene el dominio Nterminal (NTD) y un dominio de unión al receptor (RBD) que confiere el enlace al receptor huésped, mientras que la segunda cuenta con péptido de fusión (FP), región de conexión (CR), repetición heptada 1 (HR1), repetición heptada 2 (HR2) y hélice central ( $\mathrm{CH})$, que, en general, permiten la fusión de membranas, el proceso esencial para la entrada del virus. ${ }^{7,8,9,10}$ Además, produce 16 proteínas no estructurales y nueve accesorias que podrían jugar un papel importante contra la inmunidad del huésped. ${ }^{10}$ 


\section{Fisiopatología de la COVID-19}

La proteína $S$ presente en la envoltura viral del SARS-CoV-2 es capaz de reconocer a la Enzima Convertidora de Angiotensina-2 (ECA-2). Esta es una carboxipeptidasa presente en la superficie celular que escinde la Angiotensina-I en Angiotensina 1-9 y la Angiotensina-2 en Angiotensina-1-7, desencadenando una respuesta vasodilatadora, antihipertrofica, proapoptótica y antiproliferativa que se opone a los efectos de la Enzima Convertidora de Angiotensina (ECA). La ECA-2 predomina en células epiteliales alveolares, riñón, hígado, cerebro, placenta, corazón, endotelio vascular e intestino. ${ }^{11,12,13,14}$

La unión de la ECA-2 a la región RBD de la proteína $S$ provoca la activación de la proteasa de serina transmembrana tipo 2 (TMPRSS2), presente en la membrana celular, la cual inducirá un cambio conformacional de la proteína $S$, permitiendo la entrada a la célula huésped y la liberación de la nucleocápside viral. De inmediato, empezará la traducción del material genético. El ARN genómico se traduce en poliproteínas replicasas virales ppla y $1 \mathrm{ab}$, que serán clivadas en productos proteicos más pequeños para inducir la formación de vesículas de doble membrana, lugar en donde podrán anclarse complejos de replicación y transcripción viral. Después, a partir de la polimerasa viral, se obtendrá ARNm subgenómico que se traducirá en proteínas estructurales y accesorias. Finalmente, el virión es ensamblado en el Retículo Endoplasmático y el Aparato de Golgi para, posteriormente, ser liberado fuera de la célula. ${ }^{7,14}$

Una vez reconocido el patógeno, se activará la cascada de señalización y se expresará interferón tipo I (IF-I) y otras citocinas proinflamatorias como parte de la primera línea de defensa. Posteriormente, los patrones moleculares activados asociados al daño serán reconocidos por células epiteliales, endoteliales y macrófagos alveolares que inducirán la liberación de citocinas y quimiocinas proinflamatorias, atrayendo al sitio de infección a macrófagos, monocitos y células T. Eventualmente, se verá comprometida la integridad de la barrera epitelial, habrá edema, infiltración celular y exfoliación de células epiteliales alveolares. Estas medidas provocarán una tormenta de citoquinas que inducirá inflamación pulmonar descontrolada. Las citocinas que predominan son la interleucina (IL) 1, IL-6, IL-10, proteína quimioatrayente de monocitos 1 (MCP1), factor de necrosis tumoral- $\alpha$ (TNF- $\alpha$ ) y el factor estimulante de colonias de granulocitos (G-CSF). Algunas de estas citocinas inducen la alteración y desprendimiento de la ECA-2; esta pérdida funcional altera la regulación de angiotensina-2, aumenta la permeabilidad vascular y la inflamación pulmonar. ${ }^{15,16}$

La respuesta inflamatoria aumentará la apoptosis de linfocitos, produciendo una marcada linfopenia, aunado a un engrosamiento de la pared alveolar por células monoclonales y macrófagos. Se ha encontrado monocitos inflamatorios específicos CD14+ y CD16+ que aumentan los niveles de IL-6, induciendo una reacción inflamatoria sistémica. Posteriormente, puede presentarse edema pulmonar con formación de membrana hialina, iniciando el síndrome de dificultad respiratoria agudo que podría agravarse a insuficiencia multiorgánica." 15,17 


\section{Etapas del desarrollo de nuevas vacunas}

El proceso que involucra desarrollar y elaborar una nueva vacuna puede tardar entre cinco y 15 años y costar más de USD $\$ 500$ millones. Las vacunas deben cumplir con los más altos estándares de seguridad y calidad, lo que ocasiona que exista un elevado índice de fracasos en las diferentes etapas del proceso y que pocas vacunas planteadas en la etapa de investigación básica lleguen a ser comercializadas. Por ejemplo, desde comienzos de la pandemia por COVID-19 se registraron más de 200 vacunas, de las cuales actualmente 21 vacunas se encuentran en fase III de la etapa de ensayos clínicos. Es por ello que el desarrollo de una vacuna, sigue una meticulosa secuencia lineal y sistemática constituida por 3 etapas, como se muestra en la tabla 1. ${ }^{1,18,19}$

Tabla 1. Etapas del desarrollo de una nueva vacuna

\begin{tabular}{|c|c|c|}
\hline Etapa & Objetivo & Procesos que se realizan \\
\hline $\begin{array}{l}\text { Exploratoria o de } \\
\text { investigación } \\
\text { básica }\end{array}$ & $\begin{array}{l}\text { Justificar el proceso } \\
\text { particular mediante el } \\
\text { cual se desarrollará la } \\
\text { vacuna con base en } \\
\text { sustentos científicos. } \\
\text { Comprende desde el } \\
\text { nacimiento teórico del } \\
\text { producto hasta la } \\
\text { prueba de concepto }\end{array}$ & $\begin{array}{l}\text { - Planteamiento de recursos materiales, técnicos y } \\
\text { biológicos } \\
\text { - Elección de los antígenos y adyuvantes más } \\
\text { adecuados } \\
\text { - Elección de modelos animales y la vía de } \\
\text { administración } \\
\text { - Evaluación de la cobertura poblacional e interferencia } \\
\text { con otras patentes } \\
\text { - Evaluación de inmunigenicidad y toxicidad preliminar } \\
\text { - Estudios sobre el mecanismo de acción y } \\
\text { caracterización de la respuesta inmune } \\
\text { - Pruebas de concepto }\end{array}$ \\
\hline $\begin{array}{l}\text { Preclínica o de } \\
\text { desarrollo } \\
\text { preclínico y } \\
\text { farmacéutico }\end{array}$ & $\begin{array}{l}\text { Valorar los efectos de } \\
\text { una vacuna en } \\
\text { animales (ratones, } \\
\text { monos, conejos) y } \\
\text { modelos análogos al } \\
\text { cuerpo humano } \\
\text { (cultivos de tejidos o } \\
\text { células) }\end{array}$ & 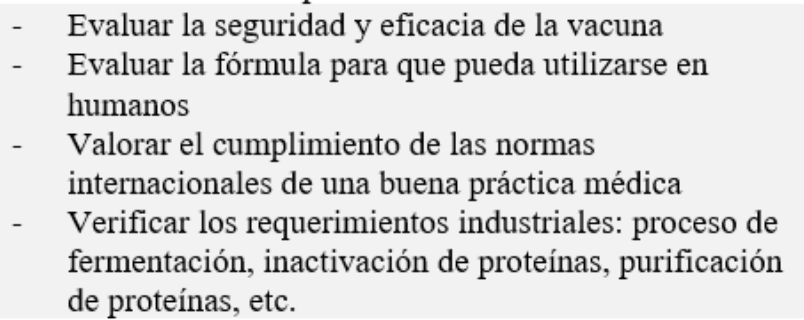 \\
\hline $\begin{array}{l}\text { Etapa clínica o de } \\
\text { ensayos clínicos }\end{array}$ & $\begin{array}{l}\text { Evaluar los efectos que } \\
\text { produce una vacuna en } \\
\text { humanos }\end{array}$ & $\begin{array}{l}\text { - Valorar la inmunogenicidad, datos de toxicidad y } \\
\text { posible impacto en la salud pública } \\
\text { - Evaluar la aceptación en la comunidad, rentabilidad y } \\
\text { perfil de riesgo-beneficio } \\
\text { - Evaluar la eficacia y seguridad }\end{array}$ \\
\hline
\end{tabular}

Características generales de las diferentes etapas para el desarrollo de una nueva vacuna ${ }^{18,19,20}$

Aquellas vacunas que hacen la transición del laboratorio a los ensayos clínicos, se enfrentarán a las cuatro fases descritas en la tabla 2. ${ }^{19,20}$ 
Tabla 2. Fases de la etapa de ensayos clínicos

\begin{tabular}{|c|c|c|c|}
\hline $\begin{array}{l}\text { Fase de } \\
\text { ensayos } \\
\text { clinicos }\end{array}$ & Tipo de ensayo & Población & Objetivos \\
\hline Fase I & $\begin{array}{l}\text { Ensayos de etiqueta } \\
\text { abierta y no } \\
\text { aleatorizados, aunque } \\
\text { pueden ser ensayos } \\
\text { controlados } \\
\text { aleatorios }\end{array}$ & $\begin{array}{l}\text { Menos de } 100 \text { adultos } \\
\text { humanos sanos, con } \\
\text { bajo riesgo de infección } \\
\text { e inmunocompetentes } \\
\text { sin experiencia }\end{array}$ & $\begin{array}{l}\text { Su objetivo primario es la evaluación de la } \\
\text { seguridad y reactogenicidad de la vacuna, } \\
\text { dejando como objetivo secundario la } \\
\text { recopilación de información respecto a la } \\
\text { eficacia. También se valora la dosis más } \\
\text { adecuada, esquema de vacunación y modo } \\
\text { de administración }\end{array}$ \\
\hline Fase II & $\begin{array}{l}\text { Ensayos controlados } \\
\text { aleatorizados, pueden } \\
\text { ser simple o doble } \\
\text { ciego. Se realizan } \\
\text { estudioso de desafio }\end{array}$ & $\begin{array}{l}\text { De } 200 \text { a } 500 \\
\text { voluntarios, puede } \\
\text { incluir adultos, } \\
\text { adolescentes, niños, } \\
\text { lactantes hasta mujeres } \\
\text { embarazadas }\end{array}$ & $\begin{array}{l}\text { Evaluar su eficacia protectora ante la } \\
\text { enfermedad y su impacto frente a variables } \\
\text { como la edad, etnia, género y presencia de } \\
\text { anticuerpos maternos. También evalúa la } \\
\text { preparación de la vacuna, edad mínima que } \\
\text { la recibirá, dosis óptima, número de dosis, } \\
\text { secuencia e intervalo entre dosis, duración } \\
\text { de la inmunidad y aspectos cualitativos de } \\
\text { la respuesta inmune }\end{array}$ \\
\hline Fase III & $\begin{array}{l}\text { Ensayos controlados } \\
\text { aleatorizados }\end{array}$ & $\begin{array}{l}\text { Miles de individuos de } \\
\text { uno o varios países, se } \\
\text { procura involucrar a una } \\
\text { población diversa }\end{array}$ & $\begin{array}{l}\text { Evaluar la seguridad y eficacia de la } \\
\text { vacuna, entiéndase por eficacia el } \\
\text { porcentaje de reducción en la incidencia de } \\
\text { la enfermedad o infección entre los } \\
\text { vacunados }\end{array}$ \\
\hline Fase IV & $\begin{array}{l}\text { Se recaba } \\
\text { información } \\
\text { poscomerciali-zación }\end{array}$ & $\begin{array}{l}\text { Cientos de miles a } \\
\text { millones de personas }\end{array}$ & $\begin{array}{l}\text { Seguir evaluando la efectividad y seguridad } \\
\text { de la misma en un ambiente clínico diario o } \\
\text { cotidiano }\end{array}$ \\
\hline
\end{tabular}

Características generales de cada una de las 4 fases que involucra la etapa de ensayos clínicos en el desarrollo de una nueva vacuna $18,19,20$

Normalmente, si los resultados arrojados en la fase III son satisfactorios, el fabricante de la vacuna puede proceder a presentar una solicitud a la autoridad reguladora de cada país para la autorización y comercialización sel producto en dicha nación. Actualmente, a causa de la pandemia por la COVID-19 es esencial hacer llegar a la población una vacuna rápidamente, lo que abre la oportunidad a que las autoridades respectivas otorguen la llamada autorización de uso de emergencia, que consiste en un mecanismo que facilita el uso y disposición de contramedidas médicas en situaciones de emergencia de salud pública. ${ }^{18,19,20}$ 
Tabla 3. Vacunas para COVID-19 que se encuentran en fase III

\begin{tabular}{|c|c|c|c|c|c|c|}
\hline Desarrollador y fabricante & Nombre de la vacuna & $\begin{array}{ll}\text { Plataforma de } & N \\
\text { vacuna } & d t \\
d c\end{array}$ & $\begin{array}{l}\text { No. } \\
\text { de } \\
\text { dosis }\end{array}$ & $\begin{array}{l}\text { Via de } \\
\text { administración }\end{array}$ & $\begin{array}{l}\text { Momento } \\
\text { de dosis } \\
\text { (dias) }\end{array}$ & $\begin{array}{l}\text { Ensayos clinicos de } \\
\text { fase } 3\end{array}$ \\
\hline Moderna/NIAID & mRNA-1273 & ARN & 2 & IM & 0,28 & NCT 04470427 \\
\hline $\begin{array}{l}\text { BioNTech/Fosun } \\
\text { Pharma/Pfizer }\end{array}$ & BNT 162b2/Tozinameran & ARN & 2 & IM & 0,21 & NCT 04368728 \\
\hline $\begin{array}{l}\text { University of } \\
\text { Oxford/AstraZeneca }\end{array}$ & $\begin{array}{l}\text { ChAdOx1 } \\
\text { nCoV-19/AZD12222 }\end{array}$ & $\begin{array}{l}\text { Vector viral no } \\
\text { replicable }\end{array}$ & 2 & IM & 0,28 & $\begin{array}{l}\text { ISRCTN89951424 } \\
\text { NCT } 04516746 \\
\text { NCT } 04540393\end{array}$ \\
\hline Novavax & NVX-Cov2373 & $\begin{array}{l}\text { Subunidad de } \\
\text { proteinas }\end{array}$ & 2 & IM & 0,21 & $\begin{array}{l}220-004123-16 \\
\text { NCT } 04611802\end{array}$ \\
\hline Sinovac & CoronaVac & Virus inactivado & 2 & IM & 0,14 & $\begin{array}{l}\text { NCT } 04456595 \\
\text { NCT } 04582344 \\
\text { NCT } 04617483\end{array}$ \\
\hline $\begin{array}{l}\text { Wuhan Institute of } \\
\text { Biological } \\
\text { Products/Sinopharm }\end{array}$ & Sin nombre & Virus inactivado & 2 & IM & 0,21 & $\begin{array}{l}\text { NCT } 04612972 \\
\text { ChiCTR2000034780 } \\
\text { NCT } 04612972\end{array}$ \\
\hline $\begin{array}{l}\text { Beijing Institute of } \\
\text { Biological } \\
\text { Products/Sinopharm }\end{array}$ & BBIBP-CorV & Virus inactivado & 2 & IM & 0,21 & NCT 04560881 \\
\hline $\begin{array}{l}\text { CanSino Biological } \\
\text { Inc/Beijin Institute of } \\
\text { Biotechnology }\end{array}$ & Ad5-nCov/Convidicea & $\begin{array}{l}\text { Vector viral no } \\
\text { replicable }\end{array}$ & 1 & IM & & $\begin{array}{l}\text { NCT } 04526990 \\
\text { NCT } 04540419\end{array}$ \\
\hline $\begin{array}{l}\text { Gameleya Research } \\
\text { Institute }\end{array}$ & $\begin{array}{l}\text { Gam-COVID-Vac/Sputnik } \\
\mathrm{V}\end{array}$ & $\begin{array}{l}\text { Vector viral no } \\
\text { replicable }\end{array}$ & 2 & IM & 0,21 & $\begin{array}{l}\text { NCT } 04530396 \\
\text { NCT } 04564716 \\
\text { NCT } 04642339\end{array}$ \\
\hline $\begin{array}{l}\text { Janssen Pharmaceutical } \\
\text { Companies / Johnson \& } \\
\text { Johnson }\end{array}$ & Ad26.COV2.S & $\begin{array}{l}\text { Vector viral no } \\
\text { replicable }\end{array}$ & 2 & IM & 0,56 & NCT 04535453 \\
\hline Medicago & Sin nombre & $\begin{array}{l}\text { Partículas } \\
\text { Similares al virus } \\
\text { (VLP) }\end{array}$ & 2 & IM & 0,21 & NCT 04636697 \\
\hline Bharat Biotech & BBV-152/Covaxin & Virus inactivado & 2 & IM & 0,28 & NCT 04641481 \\
\hline $\begin{array}{l}\text { Anhui Zhifei Longcom } \\
\text { Biopharmaceutical/Institute } \\
\text { of Microbiology, Chinese } \\
\text { Academy of Sciences }\end{array}$ & RBD-Dimer & $\begin{array}{l}\text { Subunidad de } \\
\text { proteinas }\end{array}$ & 3 & IM & $0,28,56$ & ChicTR2000040153 \\
\hline Curevac AG & CVnCov Vaccine & ARN & 2 & IM & 0,28 & NTC0 4674189 \\
\hline $\begin{array}{l}\text { Research Institute for } \\
\text { Biologica Safety Probems, } \\
\text { Rep of Kazakhstan }\end{array}$ & QazCovid-in & Virus inactivado & 2 & IM & 0,21 & NTC04691908 \\
\hline Zydus Cadila & ncov vaccine & $\begin{array}{l}\text { Acido } \\
\text { desoxirribonucleico } \\
\text { (ADN) }\end{array}$ & 3 & ID & $0,28,56$ & CTRI/2020/07/026352 \\
\hline Serum Institute of India & Covishield & $\begin{array}{l}\text { Vector viral no } \\
\text { replicable }\end{array}$ & 2 & IM & 0,28 & $\begin{array}{l}\text { CTRI / } \\
2020 / 08 / 027170\end{array}$ \\
\hline COVAXX & UB- 612 & $\begin{array}{l}\text { Subunidad de } \\
\text { proteinas }\end{array}$ & 2 & IM & 0,28 & NCT 04683224 \\
\hline Clover & SCB-2019 & $\begin{array}{l}\text { Subunidad de } \\
\text { proteínas }\end{array}$ & 2 & IM & 0,21 & NCT 04672395 \\
\hline AnGes & AG0302-COVID19 & $\mathrm{ADN}$ & 2 & IM & $\begin{array}{l}0,140 \\
28\end{array}$ & NCT 04655625 \\
\hline Inovio & INO -4800 & $\mathrm{ADN}$ & 2 & ID & 0,28 & NCT 04642638 \\
\hline
\end{tabular}

La fase IV de ensayos clínicos ocurre posterior a la comercialización de la vacuna y es indispensable para seguir estudiando los efectos de la vacuna, ya que podrían reportarse efectos adversos distintos a los registrados y las cifras de eficacia son más precisas. ${ }^{18,19}$

\section{Tipos de vacunas}

Generar una respuesta inmune eficaz para evitar la enfermedad o la infección de un agente patógeno mediante la exposición a este mismo, pero con modificaciones que eviten la enfermedad que este provoca, es el objetivo de todas las vacunas. La evidente necesidad de desarrollar una vacuna precozmente contra SARS-CoV-2 hizo que se plantearan una gran variedad de enfoques para diseñar y desarrollar vacunas contra este patógeno. La tabla 3 describe los tipos de 
vacunas que se encuentran en ensayos de fase III. Un dato sorprendente de esta carrera en búsqueda de la vacuna es la gran variedad de plataformas tecnológicas utilizadas para su desarrollo, mencionadas en la tabla 4. Los nuevos avances en áreas como la inmunología, genómica, inmunogenética, inmunogenómica, metabólica, bioinformática, biología estructural y otras más, han permitido el planteamiento de un nuevo paradigma en la elaboración de nuevas vacunas, lo cual representa la evolución hacia una nueva era en la vacunología. ${ }^{1,21,22,23}$

Tabla 4. Características generales de los tipos de vacunas

\begin{tabular}{|c|c|}
\hline Tipo de vacuna & Características \\
\hline $\begin{array}{l}\text { Basadas en } \\
\text { epitopos }\end{array}$ & $\begin{array}{l}\text { Se basan en la administración de epitopos específicos de la proteína S (por ejemplo, el } \\
\text { epitopo } 807-\mathrm{S}-26 \text { o el } 1140-\mathrm{S}-25 \text { ), a la cual se unen los anticuerpos, linfocitos T y B } \\
\text { para neutralizar al SARS-CoV-2 }\end{array}$ \\
\hline $\begin{array}{l}\text { De proteínas } \\
\text { recombinantes }\end{array}$ & $\begin{array}{l}\text { Es necesario tener conocimiento de la secuencia de } \mathrm{ADN} \text { que codifica para la proteína } \\
\text { estructural clave del patógeno, en el caso del SARS-CoV-2 la proteína S. Dicha } \\
\text { secuencia de } \mathrm{ADN} \text { será introducida en células para la producción de la proteína y } \\
\text { posterior purificación. Esta proteína se introducirá en el organismo para inducir la } \\
\text { respuesta inmune. Este tipo de vacunas suele requerir el uso de adyuvantes }\end{array}$ \\
\hline De vectores virales & $\begin{array}{l}\text { Son virus recombinantes o modificados con la función de transportar material genético } \\
\text { ajeno al mismo virus hacia una célula objetivo con el fin de transcribir y traducir esta } \\
\text { secuencia genética para sintetizar la proteína } \mathrm{S} \text { que el sistema inmune reconocerá para } \\
\text { así inducir la respuesta inmune. Si a los virus se les inactivan los genes de replicación } \\
\text { se dice que son no replicantes, mientras que si se conservan los genes de replicación se } \\
\text { conocen como virus replicantes }\end{array}$ \\
\hline $\begin{array}{l}\text { Basadas en ácidos } \\
\text { nucleicos }\end{array}$ & $\begin{array}{l}\text { Hay dos tipos: de ADN y ARN. Consisten en emplear instrucciones genéticas en forma } \\
\text { de } \mathrm{ADN} \text { o ARN para su introducción en las células humanas con el fin de producir la } \\
\text { proteína S de SARS-CoV-2 e inducir una respuesta inmune. A diferencia de las de } \\
\text { vectores virales, estas emplean como vector nanoparticulas lipídicas sintéticas, } \\
\text { sistemas de electroporación y nanoparticulas catiónicas }\end{array}$ \\
\hline De virus atenuados & $\begin{array}{l}\text { Se logra sembrando en múltiples cultivos de células humanas y animales al SARS- } \\
\text { CoV-2 con el objetivo de producir mutaciones que mermen su capacidad de producir } \\
\text { enfermedad. Posteriormente se prueba como vacuna }\end{array}$ \\
\hline $\begin{array}{l}\text { De virus } \\
\text { inactivados }\end{array}$ & $\begin{array}{l}\text { El SARS-CoV-2 posee su estructura externa sin modificaciones, pero es incapaz de } \\
\text { replicarse. Esto se debe a que ha sido inactivado por medio de métodos fisicos o } \\
\text { químicos. Una vez inactivado es probado como vacuna. Este tipo de vacuna } \\
\text { generalmente requiere de adyuvantes }\end{array}$ \\
\hline $\begin{array}{l}\text { Basadas en } \\
\text { partículas similares } \\
\text { a virus (VLPs) }\end{array}$ & $\begin{array}{l}\text { Se producen gracias a la capacidad de autoensamble de las proteínas estructurales del } \\
\text { SARS-CoV-2 dando lugar a las VLPs que son estructuralmente análogas, y en algunos } \\
\text { casos idénticas, al virus original. Su estructura comparable a los virus las hace } \\
\text { altamente inmunógenas y su carencia de genoma inhibe la posibilidad de reversión a } \\
\text { virulencia }\end{array}$ \\
\hline
\end{tabular}

Características generales de los diferentes tipos de vacunas mayormente empleadas en la carrera por el desarrollo de la vacuna contra la COVID-1919, 21, 22, 23, 24, 2526

\section{BNT162b2, la vacuna de Pfizer y BioNTech contra la COVID-19}

BNT162b2 fue la primera vacuna contra la COVID-19 aprobada por la FDA. Requiere un almacenamiento a temperaturas de ultracongelación $\left(-70 \mathrm{a}-80^{\circ} \mathrm{C}\right)$ debido a que está compuesta por ARNm, el cual codifica al dominio RBD de la proteína $S$ del SARS-CoV-2 y está encapsulado por nanopartículas lipídicas (mezcla de grasas especializadas, colesterol y polietilenglicol) que permiten al ARNm llegar a las células del cuerpo después de la administración de la vacuna. 
Una vez dentro de la célula huésped, el $A R N m$ inicia la síntesis de la proteína $S$ que activará una respuesta inmune humoral y de células T. 4, 27, 28, 29, 30

Hallazgos preliminares del ensayo clínico de fase III, indican que la vacuna BNT162b2 fue un 95\% efectiva para prevenir la COVID-19 sintomática, confirmado por laboratorio en personas sin evidencias de infección previa por SARS-CoV-2. Se encontró también una eficacia constante de 92\%, aproximadamente, en diversas categorías: edad, sexo, raza, etnia y en personas con comorbilidades. ${ }^{27,31,32}$

Los informes indican que el $84.7 \%$ de los vacunados presentó, por lo menos, una reacción local, siendo el dolor la más frecuente, seguido de hinchazón y enrojecimiento. Así mismo, el $77.4 \%$ de los vacunados informó al menos una reacción sistémica; las más comunes fueron: fatiga, cefalea, mialgias y fiebre. Ambos tipos de reacciones se exacerbaron después de la segunda dosis, pero se resolvían aproximadamente en un día. Las reacciones sistémicas se reportaron mayormente en grupos de edad joven en comparación con los de edad avanzada. ${ }^{27,31,33}$

Se han reportado reacciones alergias graves en individuaos vacunados, y se atribuyen dichas respuestas alérgicas al polietilenglicol presente en la vacuna. Algunos casos reportados indican los siguientes signos y síntomas: disnea, taquicardia, erupciones cutáneas, edema palpebral y de lengua, mareos y ronquera. $^{28,34}$

\section{mRNA-1273, la vacuna de Moderna}

Fue la segunda vacuna de ARNm que recibió aprobación en Estados Unidos y Canadá, demostrando una efectividad del $94.1 \%$ y alta capacidad para prevenir la enfermedad grave. Utiliza ARNm cubierto por nanopartículas de lípidos que expresa antígeno $S-2 \mathrm{P}$, capaz de traducir la proteína $S$ del SARS-CoV-2, estabilizada y previa a la perfusión. De esta forma, al reconocer el antígeno, el huésped desarrollará una respuesta inmunológica capaz de neutralizar la proteína S. ${ }^{35,36,37}$

Las reacciones adversas locales fueron consideradas de grado leve. El síntoma predominante fue dolor, y en algunos participantes se desarrollaron reacciones tardías (eritema e induración), resolviéndose en cuatro a cinco días. Los eventos sistémicos se consideraron de grado moderado a grave, con aumento de severidad después de la segunda dosis, observándose fatiga, artralgia, mialgia y cefalea en $50 \%$ de los participantes. Estas reacciones fueron transitorias, resolviéndose dos días después. También se registraron reacciones de hipersensibilidad en 1.5\% de los aspirantes, y tres participantes presentaron parálisis de Bell. ${ }^{36,38,39}$

\section{ChAdOx1 nCoV-19, la vacuna de AstraZeneca y la Universidad de Oxford}

El 4 de enero de 2021, la COFEPRIS aprobó para uso de emergencia la vacuna ChAdOx 1 nCoV-19. ${ }^{40,41}$ 
Se basa en un vector adenoviral de chimpancé de replicación deficiente, que procede del serotipo Y25 de adenovirus de simio, el cual alberga el gen que codifica a la proteína $S$ de longitud completa del SARS-Cov-2 y posee una secuencia líder del activado tisular del plasminógeno. Una vez adentrado el vector adenoviral en las células huésped, estas sintetizaran proteína $S$ para inducir la producción de anticuerpos y la activación de inmunidad celular contra esta proteína, bloqueando la entrada viral a las células huésped impidiendo la infección. ${ }^{42,43,44,45}$

Para evaluar su seguridad y eficacia se realizaron cuatro ensayos clínicos. Los resultados revelaron que ChAdOx1 nCoV-19 tiene una eficacia del 70.4\% posterior a dos dosis y del $64.1 \%$ después de una dosis. ${ }^{42,46}$

De manera general, se observó un buen perfil de seguridad, donde el dolor y la sensibilidad en la zona de vacunación fueron los efectos adversos locales más presentados y se dieron mayormente durante las 48 horas siguientes a la vacunación; no hubo efectos adversos locales graves. Por otro lado, las mialgias, cefaleas, fatiga y fiebre fueron las reacciones adversas sistémicas más reportadas. Se observó una reducción en la reactogenicidad después de la aplicación de la segunda dosis en comparación con la primera, y los adultos mayores presentaron menor reactogenicidad que cualquier otro grupo poblacional. ${ }^{42,43,44}$

Igualmente, se reportó un caso de fiebre mayor a $40^{\circ} \mathrm{C}$, el cual se recuperó rápidamente y no necesito hospitalización. Y se informó de la aparición de eventos tromboembolicos asociados a la vacunación; sin embargo, la tasa de incidencia fue mucho menor de la esperada. ${ }^{42,47}$

\section{Gam-COVID-Vac (Sputnik V), la vacuna rusa contra la COVID-19}

Es una vacuna vectorial heteróloga que utiliza adenovirus recombinantes de tipo $26(\mathrm{rAd} 26)$ y 5 (rAd5) como vehículos para expresar la proteína $S$ de cadena larga de SARS-CoV-2, permitiendo desarrollar inmunogenicidad bidireccional capaz de neutralizar no solo el antígeno objetivo, sino también el factor vectorial. ${ }^{48,49,50}$

Cuenta con dos presentaciones, congelada (Gam-COVID-Vac) y liofilizada (Gam-COVIDVac-Lyo), y su empleo fue tema de controversia por algunos meses, debido a una carencia de información de acceso público sobre los estudios que validaran su administración. Las dudas fueron disipadas con la publicación del ensayo de fase III, que evidenciaba una efectividad del 91.6\% 21 días después de haberse administrado la primera dosis. También mostraba datos preliminares acerca de un 100\% de eficacia en la prevención de COVID-19 grave en individuos que recibieron las dos dosis. ${ }^{49,50}$

Los ensayos no registraron reacciones adversas que pusieran en riesgo la vida. La mayoría de las alteraciones relacionadas con la vacuna se clasificaron en grado 1, predominando dolor en el área de inyección, astenia, fiebre, mialgias, artralgias y cefalea. ${ }^{49,50}$ 


\section{Ad5-nCoV, la vacuna china desarrollada por CanSino y el Instituto de Biotecnología de Beijing}

Es una vacuna monodosis y ha consolidado aprobación de emergencia en China, México y Pakistán. ${ }^{51,52}$

Utiliza adenovirus tipo 5 (Ad5) con un defecto que impide su replicación E1/ E3. Además, posee dos genes específicos que confieren la base para la formación de inmunogenicidad: el que codifica la proteína $S$ y el de señal activadora de plasminógeno tisular. Es así como la elevada expresión de la proteína estructural de SARS-CoV-2 permite que el sistema inmunológico desarrolle una respuesta específica que inhibe la replicación del virus. ${ }^{53}$

Mostro un nivel de eficacia del $65.7 \%$. Sin embargo, uno de sus grandes problemas está relacionado con la inmunidad prexisten en grupos poblacionales, debido a un alto nivel de exposición al adenovirus, ya sea de forma natural o por la aplicación de vacunas. Esto se observó en el ensayo de fase II, donde 266 participantes presentaron inmunidad alta y 242 inmunidad baja hacia el Ad5 antes de aplicar la vacuna. Los anticuerpos neutralizantes frente al virus eran más altos en individuos con baja inmunidad que aquellos con inmunogenicidad alta preexistente. Particularmente, la disminución de la potencia fue registrada en mayores de 54 años. Esto pone en duda la eficacia vacunal hacia estos grupos, por lo que se evalúa una segunda dosis para compensar y aumentar los niveles de anticuerpos neutralizantes. ${ }^{51}$

En general, las reacciones adversas fueron leves o moderadas (dolor local en el área de inyección, fatiga, fiebre y dolor de cabeza). Solo en 20 participantes se registraron reacciones de grado 3 , principalmente fiebre, y se resolvieron sin medicación a las $72-92$ horas. $^{51,52}$

\section{CoronaVac, la vacuna de Sinovac}

A partir de muestras de lavado broncoalveolar de pacientes hospitalizados por COVID-19, se aisló la cepa CN02, identificada por su capacidad de desarrollar enfermedad grave, con el fin de crear una vacuna inactivada. ${ }^{54,55,56}$

Actualmente se cuenta con dos ensayos clínicos que ponen de manifiesto su eficacia. Cada uno dividido en dos fases con el fin de evaluar parámetros de seguridad y eficacia ante determinadas características; por ejemplo, dosificación $(1.5 \mu \mathrm{g}, 3 \mu \mathrm{g}$ y $6 \mu \mathrm{g})$, intervalo de días entre aplicación (14 y 28) y grupos de edad (18 a 59 años y 60 años o más)..$^{54,56}$

Gracias a los resultados obtenidos se reconocieron tres puntos. En primer lugar, se estableció la necesidad de dos dosis con un intervalo entre ellas de 28 días, debido a que fue hasta después de completar este esquema que se observó el aumento de citocinas Th2 y linfocitos $\mathrm{B}$, aunado a una disminución de linfocitos T citotóxicos CD8+. En segundo lugar, el título medio geométrico -GMTpara anticuerpos neutralizantes (23.8 y 65.4) presentado es suficiente para crear inmunogenicidad satisfactoria. Y, en tercer lugar, las dosis de $3 \mu \mathrm{g}$ y $6 \mu \mathrm{g}$ ofrecen una protección inmune similar. ${ }^{54,56}$

En general, las reacciones adversas como dolor local relacionado con el sitio de inyección, fiebre y cefalea, se resolvieron en 48 horas. Solo se observó una 
reacción considerada como grave en el ensayo correspondiente al grupo de 18 a 59 años, fase 1, dosificado con $6 \mu \mathrm{g}$; se registró un caso (de 24 participantes) de hipersensibilidad con presencia de urticaria aguda después de la primera dosis. ${ }^{54}$, 56

\section{Janssen y Johnson \& Johnson presentan su vacuna Ad26.COV2.S}

Utiliza al adenovirus tipo 26, en el cual se implanta un gen que expresa la proteína $S$ del coronavirus capaz de activar al sistema inmunológico. Empezará a producir anticuerpos y demás células inmunes específicas que reconocerán y neutralizarán la proteína $S$. De esta forma, si alguna persona vacunada se expone al SARS$\mathrm{CoV}-2$, esta va a tener la capacidad de inhibir su replicación. ${ }^{57,58}$

Resultados preliminares del ensayo clínico de fase I-IIa arrojaron que la vacuna posee una eficacia del $66.3 \%$ para prevenir la enfermedad provocada por SARSCoV-2, observándose seroconversión en $100 \%$ de los participantes después del día 57, acompañado de un modesto incremento en la actividad de células Th1 y T CD $8+.^{57}$

Los reportes arrojaron tres puntos clave: la frecuencia de eventos adversos era mayor en participantes que recibieron dosis altas, los adultos mayores revelaron menos reacciones (mayormente de grado 3) que los grupos jóvenes y, en comparación con las demás vacunas, se presentó menor incidencia y gravedad de eventos adversos ante la aplicación de la segunda dosis. Las reacciones locales fueron leves: dolor en el lugar de aplicación, cefalea, fatiga y mialgia. Pocos participantes registraron fiebre de 39.0 a $40.0^{\circ} \mathrm{C}$, la cual fue remitida con uso de antipiréticos. ${ }^{57}$

La tabla 5 describe los costos de las vacunas, así como su temperatura de conservación.

Tabla 5. Precio y temperatura de conservación de las vacunas aprobadas

\begin{tabular}{ccc}
\hline Vacuna & $\begin{array}{c}\text { Costo aproximado en dólares } \\
\text { (por dosis) }\end{array}$ & $\begin{array}{c}\text { Conservación } \\
\left({ }^{\circ} \text { C) }\right.\end{array}$ \\
BNT162b2 & 19 a 21 & -80 a -60 \\
mRNA-1273 & 25 a 37 & 2 a 8 \\
ChAdOx1 & 2.4 a 4.5 & 2 a 8 \\
nCoV-19 & 10 a 20 & 2 a 8 \\
Gam-COVID- & 4 & 2 a 8 \\
Vac & 15 a 30 & 2 a 8 \\
Ad5-nCoV & 10 a 20 & 2 a 8 \\
CoronaVac & & \\
Ad26.COV2.S &
\end{tabular}

Se muestra el precio aproximado de las diferentes vacunas aprobadas por la COFEPRIS, FDA y EMA, así como la temperatura a la que deben ser transportadas para mantenerlas funcionales. 


\section{Discusión}

Cada vacuna demostró tener una eficacia distinta, pero solo tres obtuvieron una eficacia superior al 90\%: BNT162b2, mRNA-1273 y Gam-COVID-Vac, siendo la primera la de mayor eficacia reportada hasta el momento, con un 95\%. Todas las vacunas mostraron una eficacia igual o mayor al 50\%; solo la CoronaVac registró la menor de todas, con un 50\%. ${ }^{31,32,38,39,49,54,56}$

Mayormente, se presentaron reacciones adversas locales y sistémicas de leves a moderadas, es decir dolor, hinchazón y enrojecimiento en el sitio de punción, así como fatiga, cefalea, fiebre y mialgias. Sin embargo, algunas vacunas presentaron reacciones adversas inesperadas: algunas personas que recibieron BNT162b2 presentaron reacciones alérgicas; mRNA-1273 reportó reacciones de hipersensibilidad y parálisis de Bell; Ad5-nCoV y CoronaVac reportaron casos de reacciones adversas grado 3 o graves, y ChAdOx $1 \mathrm{nCoV}-19$ fue la más polémica, reportando múltiples reacciones adversas inesperadas (fiebre mayor a $40^{\circ} \mathrm{C}$ y tromboembolismo). ${ }^{31,33,38,39,42,47,51,52,54,56}$

Todas las vacunas se evaluaron en una población mayor a 17 o 18 años tanto en hombres como mujeres, ningún estudio incluyo menores de esta edad. También se excluyó a embarazadas e inmunocomprometidos y, en algunos casos, se incluyó a pacientes con comorbilidades como diabetes e hipertensión. Se ha optado por administrar las vacunas a la población general mayor de 17 años, incluyendo embarazadas, inmunocomprometidos y con comorbilidades, dejando de lado la población pediátrica, la cual es un importante foco para la diseminación de la infección y pudiera retrasar el alcance de la inmunidad de rebaño. ${ }^{31,32,33,38,39,42,}$ $49,51,52,54,55,56,57$

Todas las vacunas analizadas se administran por vía intramuscular, solamente una es monodosis (Ad5-nCoV) y una de ellas (BNT162b2) requiere de ultracongelación $\left(-80^{\circ} \mathrm{C}\right.$ a $\left.-60^{\circ} \mathrm{C}\right)$. El precio de cada una de ellas es variable, pero la más cara reportada es la desarrollada por Moderna ( 25 y 37 dólares por dosis), mientras que la más barata pudiera ser la desarrollada por CanSinoBio ( 4 dólares por dosis), o bien la desarrollada por AstraZeneca (2.40 a 4.50 dólares por dosis). Debe tomarse en cuenta que la primera solo requiere una dosis, mientras que la segunda necesita de dos. ${ }^{31,32,33,38,39,42,49,51,52,54,55,56,57}$

Se evaluó la duración de la respuesta inmune de acuerdo con el título de anticuerpos neutralizantes que presentaba cada individuo después de un determinado tiempo, por lo que la duración de la respuesta inmune se limita a los cohortes determinados por cada estudio. De igual modo, no se sabe con precisión la eficacia de las vacunas frente a nuevas variantes del SARS-CoV-2. Actualmente se está estudiando la posibilidad de combinar diferentes vacunas, especialmente BNT162b2 con ChAdOx1 nCoV-19, que han arrojado resultados bastante prometedores, donde después de la administración de ChAdOx1 nCoV-19 se administró BNT162b2, lo cual arrojó mejores resultados que el esquema solo de

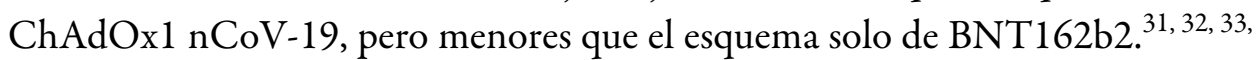
$38,39,42,49,51,52,54,55,56,57$ 


\section{Conclusión}

Determinar que una vacuna es mejor que otra, dependerá ampliamente del contexto o término bajo el que se evalúe. De este modo, la mejor vacuna en términos de eficacia es la BNT162b2; en seguridad podría ser Gam-COVIDVac; en dosis requeridas la $\mathrm{Ad} 5-\mathrm{nCoV}$, y en precio la $\mathrm{Ad} 5-\mathrm{nCoV}$ o bien la ChAdOx1 nCoV-19. En cuanto a duración de la respuesta inmune, protección contra variantes del SARS-CoV-2 y cobertura poblacional de las vacunas, hace falta realizar más estudios para delimitar la capacidad de cada vacuna en estos aspectos. Todas las vacunas, excepto BNT162b2, pueden trasportarse a entre 2 a $8^{\circ} \mathrm{C}$. Y ninguna vacuna se administra por conjuntivas. Actualmente no existe una vacuna ideal contra la COVID-19; no obstante, existen muy buenas opciones en el mercado y se recomienda a la población general vacunarse lo antes posible con la vacuna que se le ofrezca y que este autorizada para su uso por los organismos reguladores de su país. Esto con el fin de alcanzar la inmunidad de rebaño en el menor tiempo posible y así poner fin a la actual pandemia por SARS-CoV-2.

\section{Referencias}

1. Urbiztondo L, Borràs E, Mirada G. Vacunas contra el coronavirus. Vacunas. 2020; 21(1):69-72. https://doi.org/10.1016/j.vacun.2020.04.002

2. Lanata-DelasCasas CF, Gotuzzo-Herencia E. Estrategias y situación actual de la carrera para el desarrollo de vacunas contra el SARS-CoV-2. Revista Peruana de Medicina Experimental y Salud Pública. 2020; 37(3):401-402. https:// dx.doi.org/10.17843/rpmesp.2020.373.6522

3. COVID-19 Vaccine Tracker. McGill COVID19 Vaccine Tracker Team. Website: https://covid19.trackvaccines.org/vaccines/

4. Tumban E. Lead SARS-CoV-2 Candidate Vaccines: Expectations from Phase III Trials and Recommendations Post-Vaccine Approval. Viruses. 2021; 13(1) 54: 1-18. https://doi.org/10.3390/v13010054

5. Beverley P C L. Immunology of Vaccination. British Medical Bulletin. 2002; 62(1): 15-28. https://doi.org/10.1093/bmb/62.1.15

6. Alonso-Espadalé RM, Marti-Solé MC. NTP 384: la inmunización activa: una herramienta de prevención. Ministerio de Trabajo y Asuntos Sociales España. Instituto de Seguridad e Higiene en el Trabajo.

7. Shereen MA, Khan S, Kazmi A, Bashir N, Siddique R. Covid-19 infection: Origin, transmission, and characteristics of human coronaviruses. Journal of Advanced Research. 2020; 24:91-98. https://doi.org/10.1016/j.jare.2020.03.005

8. Kirtipal N, Bharadwaj S, Kang SG. From SARS to SARS-CoV-2, insights on structure, pathogenicity and immunity aspects of pandemic human coronaviruses. Infection, genetics and evolution: journal of molecular epidemiology and evolutionary genetics in infectious diseases. 2020; 85. 104502. https:// doi.org/10.1016/j.meegid.2020.104502

9. Oliva-Marín JE. SARS-CoV-2 origen, estructura, replicación y patogénesis. Alerta. 2020; 3(2):79-86. https://doi.org/10.5377/alerta.v3i2.9619

10. Dai L, Gao GF. Viral targets for vaccines against COVID-19. Nat Rev Immunol. 2021;21:73-82. https://doi.org/10.1038/s41577-020-00480-0 
11. Dong Y, Dai T, Wei Y. et al. A systematic review of SARS-CoV-2 vaccine candidates. Sig Transduct Target Ther. 2020; 5:237 https://doi.org/10.1038/ s41392-020-00352-y

12. Soler JM, Lloveras J, Batlle D. Enzima conversiva de la angiotensina 2 y su papel emergente en la regulación del sistema renina-angiotensina. Medicina Clínica. 2008; 131(6):230-236. https://doi.org/10.1157/13124619

13. Ocaranza MP, Moya J, Pinto M, Escudero N, Valenzuela F, Varas M, Godoy I, Chiong M, Lavandero S, Jalil J. Menores niveles tisulares de la enzima convertidora de angiotensina I homologa (ECA-2) y angiotensina-(1-9) están asociados a mayor remodelamiento de la pared aórtica de ratas hipertensas. Revista chilena de cardiología. 2010; 29(1):69-82. https://dx.doi.org/10.4067/ S0718-85602010000100007

14. Bourgonje AR, Abdulle AE, Timens W, Hillebrands J - L, Navis GJ, Gordijn SJ, Bolling MC, Dijkstra G, Voors AA, Osterhaus AD, van der Voort PH, Mulder DJ, van Goor H. Angiotensin - converting enzyme 2 (ACE2), SARS - CoV 2 and the pathophysiology of coronavirus disease 2019 (COVID - 19). J. Pathol. 2020; 251:228-248. https://doi.org/10.1002/path.5471

15. Tsatsakis A, Calina D, Falzone L, Petrakis D, Mitrut R, Siokas V, Pennisi M, Lanza G, Libra M, Doukas SG, Doukas PG, Kavali L, Bukhari A, Gadiparthi C, Vageli DP, Kofteridis DP, Spandidos DA, Paoliello MMB, Aschner M, Docea AO. SARS-CoV-2 pathophysiology and its clinical implications: an integrative overview of the pharmacotherapeutic management of COVID-19. Food and Chemical Toxicology. 2020; 146. 111769. https://doi.org/10.1016/ j.fct.2020.111769

16. Fu Y, Cheng Y, Wu Y. Understanding SARS-CoV-2-Mediated Inflammatory Responses: From Mechanisms to Potential Therapeutic Tools. Virol. Sin. 2020; 35:266-271. https://doi.org/10.1007/s12250-020-00207-4

17. Wiersinga WJ, Rhodes A, Cheng AC, Peacock SJ, Prescott HC. Pathophysiology, Transmission, Diagnosis, and Treatment of Coronavirus Disease 2019 (COVID-19): A Review. JAMA. 2020; 324(8):782-793. doi:10.1001/ jama.2020.12839

18. OPS/OMS: Organización Panamericana de la Salud. COID-19 Fases de desarrollo de una vacuna. Sitio web: https://www.paho.org/es/documentos/covid-19-fasesdesarrollo-vacuna

19. Guevara JV, Buelvas N, Suárez R, Gutiérrez-Peña L, Chirinos R, Pérez I, Villarreal J, Urdaneta $H$. Esfuerzos para el desarrollo de vacunas y adyuvantes. Acta Bioclínica. 2017; 8(15):259-287. http://erevistas.saber.ula.ve/index.php/ actabioclinica/article/view/9972

20. Singh K, Mehta $S$. The clinical development process for a novel preventive vaccine: An overview. J Postgrad Med. 2016; 62:4-11. DOI: 10.4103/0022-3859.173187

21. León-Lara X, Otero-Mendoza F. Desarrollo de vacunas contra COVID-19. Acta Pediátrica de México. 2020; 41(4S1):23-26. http://dx.doi.org/10.18233/ APM41No4S1ppS23-S262078

22. Sterin-Prync AE. Vacunas para SARS-CoV-2, diferentes estrategias de los desarrollos en curso. Rev. Hosp. Ital. B. Aires. 2020; 40(1):1-12.

23. González-Romo F, Picazo JJ. El desarrollo de nuevas vacunas. Enfermedades Infecciosas y Microbiología Clínica. 2015; 33(8):557-568. https:// doi.org/10.1016/j.eimc.2015.06.013 
24. Lurie N, Saville M, Hatchett R, Halton J. Developing Covid-19 Vaccines at Pandemic Speed. Journal Article. New England Journal of Medicine. 2020; 382(21):1969. https://www.nejm.org/doi/full/10.1056/NEJMp2005630

25. Blanco-Lavilla E, Bárcena-del-Riego J. Colaboraciones en ciencias de la naturaleza: vacunas basadas en cápsidas quiméricas (VLPs). Revista 100cias@uned. 2011; 4:55-61.

26. Reina J. Las vacunas de la gripe basadas en las partículas virus-like obtenidas mediante sistemas de expresión génica en células de insectos. Vacunas. 2012; 13(2):64-68. https://doi.org/10.1016/S1576-9887(12)70039-0

27. Dooling K, Marin M, Wallace M, et al. The Advisory Committee on Immunization Practices' Updated Interim Recommendation for Allocation of COVID-19 Vaccine - United States, December 2020. MMWR Morb Mortal Wkly Rep. 2021; 69:1657-1660. http://dx.doi.org/10.15585/mmwr.mm695152e2

28. Cabanillas B, Akdis C, Novak N. Allergic reactions to the first COVID - 19 vaccine: A potential role of polyethylene glycol? Allergy. 2021. https://doi.org/10.1111/ all.14711

29. Drugs and Lactation Database (LactMed) [Internet]. Bethesda (MD): National Library of Medicine (US); 2006. COVID-19 vaccines. [Updated 2020 Dec 21].

30. Le TK, Paris C, Khan KS, Robson F, Wai-Lung N, Rocchi P. Nucleic Acid-Based Technologies Targeting Coronaviruses. Trends in Biochemical Sciences. 2020. https://doi.org/10.1016/j.tibs.2020.11.010.

31. Walsh EE, Frenck RW, Falsey AR, Kitchin N, Absalon J, Gurtman A, ... Gruber WC. Safety and Immunogenicity of Two RNA-Based Covid-19 Vaccine Candidates. New England Journal of Medicine. 2020; 383(25):2439-2450. https://doi.org/10.1056/nejmoa2027906

32. Mishra SK, Tripathi T. One year update on the COVID-19 pandemic: Where are we now? Acta Tropica. 2021;214. 105778. https://doi.org/10.1016/ j.actatropica.2020.105778.

33. Centers for Disease Control and Prevention. Vaccines and Immunizations. Local Reactions, Systemic Reactions, Adverse Events, and Serious Adverse Events: Pfizer-BioNTech COVID-19 Vaccine. December 13, 2020. CDC website. https://www.cdc.gov/vaccines/covid-19/info-by-product/pfizer/ reactogenicity.html

34. Kleine-Tebbe J, Klimek L, Hamelmann E, Pfaar O, Taube C, Wagenmann M, Werfel T, Worm M. Severe allergic reactions to the COVID-19 vaccine statement and practical consequences. Allergol Select. 2021; 5(5):26-28. doi: 10.5414/ALX02215E. PMID: 33426427; PMCID: PMC7787363.

35. Anderson EJ, Rouphael NG, Widge AT, Jackson LA, Roberts PC, Makhene M, ... Beigel JH. Safety and Immunogenicity of SARS-CoV-2 mRNA-1273 Vaccine in Older Adults. New England Journal of Medicine, 2020; 383(25):2427-2438. https://doi.org/10.1056/nejmoa2028436

36. Baden LR., El-Sahly HM, Essink B, Kotloff K, Frey S, Novak R, ... Zaks T. Efficacy and Safety of the mRNA-1273 SARS-CoV-2 Vaccine. New England Journal of Medicine. 2020. 1-14. https://doi.org/10.1056/nejmoa2035389

37. Jackson LA, Anderson EJ, Rouphael NG, Roberts PC, Makhene M, Coler RN., ... Beigel JH. An mRNA Vaccine against SARS-CoV-2 - Preliminary Report. New England Journal of Medicine. 2020; 383(20), 1920-1931. https:// doi.org/10.1056/nejmoa2022483 
38. Durability of Responses after SARS-CoV-2 mRNA-1273 Vaccination. The New England Journal of Medicine. 2021; (384):80-82 DOI: 10.1056/ NEJMc2032195

39. Biomedical Advanced Research and Development Authority National Institute of Allergy and Infectious Diseases (NIAID). A Study to Evaluate Efficacy, Safety, and Immunogenicity of mRNA-1273 Vaccine in Adults Aged 18 Years and Older to Prevent COVID-19. ModernaTX, Inc. ClinicalTrials.gov Identifier: NCT04470427. https://clinicaltrials.gov/ct2/show/NCT04470427

40. ONU Noticias. COVID: La OMS recomienda la vacuna de AstraZeneca para mayores de 65 años y contra la variante de Sudáfrica. Sitio web: https:// news.un.org/es/story/2021/02/1487832

41. Gobieno de Mexico. Secretaría de Salud: Prensa. 002: Se aprueba vacuna de AstraZeneca contra COVID-19 para uso de emergencia en México. Sitio web: https://www.gob.mx/salud/prensa/002-se-aprueba-vacuna-de-astrazenecacontra-covid-19-para-uso-de-emergencia-en-mexico

42. Voysey M, Clemens SAC, Madhi SA, Weckx LY, Folegatti PM, Aley PK, ... Zuidewind P. Safety and efficacy of the ChAdOx $1 \mathrm{nCoV}-19$ vaccine (AZD1222) against SARS-CoV-2: an interim analysis of four randomised controlled trials in Brazil, South Africa, and the UK. The Lancet, 2021; 397(10269):99-111. https://doi.org/10.1016/S0140-6736(20)32661-1

43. Ramasamy MN, Minassian AM, Ewer KJ, Flaxman AL, Folegatti PM, Owens DR, ... Zizi D. Safety and immunogenicity of ChAdOx $1 \mathrm{nCoV}-19$ vaccine administered in a prime-boost regimen in young and old adults (COV002): a single-blind, randomised, controlled, phase 2/3 trial. The Lancet. 2020; 396(10267):19791993. https://doi.org/10.1016/S0140-6736(20)32466-1

44. Barrett JR, Belij-Rammerstorfer S, Dold C. et al. Phase $1 / 2$ trial of SARS$\mathrm{CoV}-2$ vaccine $\mathrm{ChAdOx} 1 \mathrm{nCoV}-19$ with a booster dose induces multifunctional antibody responses. Nat Med. 2021; 27:279-288 https://doi.org/10.1038/ s41591-020-01179-4

45. Watanabe Y, Mendonça L, Allen ER, Howe A, Lee M, Allen JD, ... Crispin M. Native-like SARS-CoV-2 spike glycoprotein expressed by ChAdOx $1 \mathrm{nCoV}-19 /$ AZD1222 vaccine. BioRxiv: The Preprint Server for Biology. 2021; 1-26. https:// doi.org/10.1101/2021.01.15.426463

46. Ewer KJ, Barrett JR, Belij-Rammerstorfer $S$. et al. T cell and antibody responses induced by a single dose of by ChAdOx1 $\mathrm{nCoV}-19$ ( AZD1222) vaccine in a phase 1/2 clinical trial. Nat Med. 2021; 27:270-278 https://doi.org/10.1038/ s41591-020-01194-5

47. Knoll MD, Wonodi C. Oxford-AstraZeneca COVID-19 vaccine efficacy. The Lancet (London, England). 2021; 397(10269):72-74. https://doi.org/10.1016/ S0140-6736(20)32623-4

48. Burki TK. The Russian vaccine for COVID-19. The Lancet. 2020; 8(11):85-86. https://doi.org/10.1016/S2213-2600(20)30402-1

49. Logunov DY, Dolzhikova IV, Shcheblyakov DV, Tukhvatulin AI., Zubkova OV, Dzharullaeva AS, ... Gintsburg AL. Safety and efficacy of an rAd26 and rAd5 vector-based heterologous prime-boost COVID-19 vaccine: an interim analysis of a randomised controlled phase 3 trial in Russia. The Lancet, 2021; 397(10275):671-681. https://doi.org/10.1016/s0140-6736(21)00234-8

50. Logunov DY, Dolzhikova IV, Zubkova OV, Tukhvatullin AI, Shcheblyakov DV, Dzharullaeva AS, ... Gintsburg AL. Safety and immunogenicity of an rAd26 and rAd5 vector-based heterologous prime-boost COVID-19 vaccine 
in two formulations: two open, non-randomised phase $1 / 2$ studies from Russia. The Lancet. 2020; 396(10255):887-897. https://doi.org/10.1016/ S0140-6736(20)31866-3

51. Zhu FC, Guan XH, Li YH, Huang JY, Jiang T, Hou LH, ... Chen W. Immunogenicity and safety of a recombinant adenovirus type-5-vectored COVID-19 vaccine in healthy adults aged 18 years or older: a randomised, doubleblind, placebo-controlled, phase 2 trial. The Lancet. 2020; 396(10249):479-488. https://doi.org/10.1016/S0140-6736(20)31605-6

52. Zhu FC, Li YH, Guan XH, Hou LH, Wang WJ, Li JX, ... Chen W. Safety, tolerability, and immunogenicity of a recombinant adenovirus type-5 vectored COVID-19 vaccine: a dose-escalation, open-label, non-randomised, first-in-human trial. The Lancet. 2020; 395(10240):1845-1854. https:// doi.org/10.1016/S0140-6736(20)31208-3

53. Feng L, Wang Q, Shan C. et al. An adenovirus-vectored COVID-19 vaccine confers protection from SARS-CoV-2 challenge in rhesus macaques. Nat Commun. 2020; 11, 4207 https://doi.org/10.1038/s41467-020-18077-5

54. Wu Z, Hu Y, Xu M, Chen Z, Yang W, Jiang Z, ... Yin W. Safety, tolerability, and immunogenicity of an inactivated SARS-CoV-2 vaccine (CoronaVac) in healthy adults aged 60 years and older: a randomised, double-blind, placebo-controlled, phase 1/2 clinical trial. The Lancet Infectious Diseases. 2021; 3099(20):1-9. https://doi.org/10.1016/s1473-3099(20)30987-7

55. Gao Q, Bao L, Mao H, Wang L, Xu K, Yang M, Li Y, Zhu L, Wang N, Lv Z, Gao H, Ge X, Kan B, Hu Y, Liu J, Cai F, Jiang D, Yin Y, Qin C, Li J,... Qin C. Development of an inactivated vaccine candidate for SARS-CoV-2. Science (New York, N.Y.). 2020; 369(6499):77-81. https://doi.org/10.1126/science.abc1932

56. Zhang Y, Zeng G, Pan H, Li C, Hu Y, Chu K, Han W, Chen Z, Tang R, Yin W, Chen X, Hu Y, Liu X, Jiang C, Li J, Yang M, Song Y, Wang X, Gao $\mathrm{Q}$, Zhu F. Safety, tolerability, and immunogenicity of an inactivated SARSCoV-2 vaccine in healthy adults aged $18-59$ years: a randomised, double-blind, placebo-controlled, phase 1/2 clinical trial. The Lancet Infectious Diseases. 2021; 21(2):181-192. https://doi.org/10.1016/S1473-3099(20)30843-4

57. Sadoff J, Le-Gars M, Shukarev G, Heerwegh D, Truyers C, de-Groot AM, Stoop J, Tete S, Van-Damme W, Leroux-Roels I, Berghmans PJ, Kimmel M, Van-Damme P, de-Hoon J, Smith W, Stephenson KE, De-Rosa SC, Cohen KW, McElrath MJ, Cormier E, ... Schuitemaker H. Interim Results of a Phase 1-2a Trial of Ad26.COV2.S Covid-19 Vaccine. The New England Journal of Medicine, NEJMoa2034201. Advance online publication. 2021. https://doi.org/10.1056/ NEJMoa2034201

58. Solforosi L, Kuipers H, Huber SKR, van-der-Lubbe JEM, Dekking L, CzapskaCasey DN, Izquierdo-Gil A, Baert MRM, Drijver J, Vaneman J, van-Huizen E, Choi Y, Vreugdenhil J, Dalebout TJ, Myeni SK, Kikkert M, Snijder EJ, Barouch DH, Koopman G, Mooij P, Bogers WMJM, Muchene L, Tolboom JTBM, Roozendaal R, Schuitemaker H, Wegmann F, Zahn RC. Immunogenicity of one- and two-dose regimens of the Ad26.COV2.S COVID-19 vaccine candidate in adult and aged rhesus macaques bioRxiv 2020.11.17.368258. https://doi.org/10.1101/2020.11.17.368258 